\title{
Multiple Sequence Alignment of the M Protein in SARS-Associated and Other Known Coronaviruses
}

\author{
SHI Ding-Hua (史定华)，ZHOU Hui-Jie(周晖杰)，WANG Bin-Bin(王斌宾)， \\ GU Yan-Hong (顾燕红), WANG Yi-Fei (王買飞) \\ Department of Mathematics, Shanghai University, Shanghai 200436, China
}

\begin{abstract}
In this paper, we report a multiple sequence alignment result on the basis of 10 amino acid sequences of the $M$ protein, which come from different coronaviruses (4 SARS-associated and 6 others known). The alignment model was based on the profile HMM (Hidden Markov Model), and the model training was implemented through the SAHMM (Self-Adapting Hidden Markov Model) software developed by the authors.
\end{abstract}

Key words SARS (Severe Acute Respiratory Syndrome), coronavirus, M (Membrane or Matrix) protein, multiple sequence alignment, profile HMM.

MSC $200060 \mathrm{~J} 20,92 \mathrm{C} 40$

\section{Introduction}

SARS is the first newly identified serious infectious disease that human being is facing at the beginning of the 21 st century. It has been primarily recognized that a variant of virus from the coronavirus family might be the candidate pathogen of SARS, as reported by WHO (World Health Organization) on April 29 , 2003 (http: //www. who. int/csr/sarsco untry/en).

Coronaviruses were first isolated from chickens in 1937. There are now approximately 15 species in this family. Coronavirus particles are irregularly shaped, round about $60-220 \mathrm{~nm}$ in diameter, with an outer envelope bearing distinctive, 'club-shaped' peplomers (round about $20 \mathrm{~nm}$ long $\times 10 \mathrm{~nm}$ at wide distal end ${ }^{[1]}$. This 'crown-like' appearance (Latin, corona) gives the family its name.

The genome size of SARS-associated coronaviruses (isolate BJ01) is $29725 \mathrm{~kb}$ and has 11 ORFs (Open Reading Frames). The whole genome is composed of a stable region encoding an RNA-dependent RNA poly-

Received May 22, 2003

Project supported by the National Natural Science Foundation of China (Grant No. 70171059 ) and the 863 Project (Grant No. 2002AA234021)

SHI Ding-Hua, Prof., E-mail: shidh2001@263.net merase (composed of 2 ORFs) and a variable region representing 4 CDSs (Coding Sequences) viral structural genes (the S, E, M, N proteins) and 5 PUPs (Putative Uncharacterized Proteins) ${ }^{[2]}$. Its gene order is identical to that of other known coronaviruses.

The S (Spike) protein, the N(Nucleocapsid) protein and perhaps together with the $M$ protein appear to be the most important candidates for the future diagnostic testing, preventing and treatment based on antibodies and vaccines, as well as exploring the immunoreactions $^{[2]}$. Due to the limit of page space, we choose the $\mathrm{M}$ protein as an illustrated example here. The $M$ protein with transmembrane-budding and envelope formation was predicted to be a mid-sized protein (221 acid amino residues). It was located at the nucleotide position 26379-27044 (isolate BJ01) ${ }^{[2]}$.

For the $M$ protein, by using the Blast method and the ClustalW 1.8 software (http:// www. ddbj. nig. ac. jp/E-mail/ clustalw-e. htm), the results on both the pair and the multiple sequence alignments have been respectively obtained and reported in literature. However, as far as the authors know, the multiple sequence alignment result based on the profile HMM has not been seen yet.

In this paper, we report some results about a multiple sequence alignment on the basis of 10 amino acid 
sequences of the $\mathrm{M}$ protein, which come from different coronaviruses in NCBI databases (http://www. ncbi.nlm. nih. gov). They covered 4 SARS-associated coronaviruses isolated from patients in Canada, USA, and China (Beijing, Hong Kong), and 6 others: 2 from human being (229E, Transmissible gastroenteritis), 3 from house animals (Porcine, Bovine, Turkey), and 1 from bird (Avian).

\section{Model and Method}

The alignment model is based on the profile HMM, and its topology as follows ${ }^{[3]}$ :

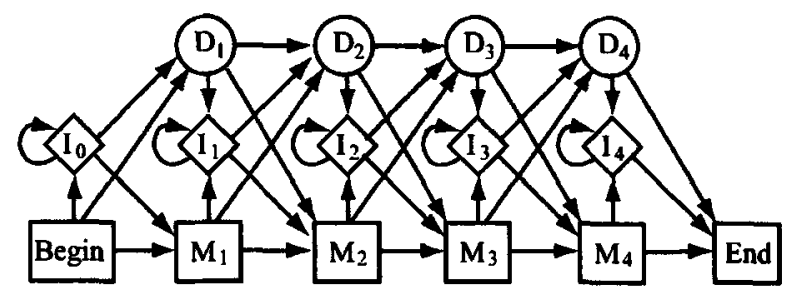

Fig.1 Topology of the profile HMM

The model training is implemented through the SAHMM software developed by the authors. The SAHMM software includes a two-stage alternative optimization method to maximize Bayesian posterior probabilities of parameters and topology for a hidden Markov model.

Let $M_{N}$ denote the profile HMM with $N$ main states, $\lambda_{N}$ the parameter set of the profile HMM (including the state transition probabilities and the symbol emission probabilities), $O=\left\{O^{(w)}\right\}, w=1,2, \cdots$ $W$, the training sequence set, and $T_{w}$ the length of the training sequence $O^{(w)}$.

The first step of two-stage alternative optimization method in the SAHMM software is parameter estimation that is to find $\lambda_{N}^{*}$ as the number of main states $N$ is fixed. By using the Bayes formula, we have

$$
\begin{aligned}
\lambda_{N}^{*} & =\arg \max _{\lambda_{N}} P\left(\lambda_{N} \mid O, M_{N}\right) \\
& \propto \arg \max _{\lambda_{N}} P\left(O \mid \lambda_{N}, M_{N}\right) P\left(\lambda_{N} \mid M_{N}\right)
\end{aligned}
$$

in which $P\left(O \mid \lambda_{N}, M_{N}\right)$ is the likelihood function of the training sequence set $O, P\left(\lambda_{N} \mid M_{N}\right)$ is the prior distribution of the parameter set $\lambda_{N}$. We use Bayesian Baum-Welch algorithm plus simulated annealing to estimate the parameters $\lambda_{N}$ of the profile HMM. The Baum-Welch algorithm is a variation of the more general EM algorithm. It iterates between an expectation step (E-step) and a maximization step (M-step). The iterative process continues until some stop rule is satisfied.

The second step of two-stage alternative optimization method in the SAHMM software is the topology optimization that is to find the following $M_{N}{ }^{*}$.

$$
\begin{aligned}
M_{N}^{*} & =\arg \max _{M_{N}} P\left(M_{N} \mid O\right) \\
& \propto \arg \max _{M_{N}} P\left(O \mid M_{N}\right) P\left(M_{N}\right)
\end{aligned}
$$

in which $P\left(M_{N}\right)$ is the prior distribution of the model topology $M_{N}$. Under the assumption of a non-information prior distribution, we have

$$
\begin{aligned}
& P\left(M_{N} \mid O\right) \propto P\left(O \mid M_{N}\right) \\
& \quad=\int P\left(O \mid M_{N}, \lambda_{N}\right) P\left(\lambda_{N} \mid M_{N}\right) \mathrm{d} \lambda_{N}
\end{aligned}
$$

Usually, the integral in Eq. (3) is difficult to calculate directly. Hence we use Bayesian Information Criterion (BIC) $)^{[4]}$ to approximate it:

$$
B I C=-2 \log P\left(O \mid \lambda_{N}^{*}, M_{N}\right)+K_{N} \log W
$$

where $K_{N}$ is the number of free parameters in the profile HMM with $N$ main states, $W$ is the sample size, and $-\log P\left(O \mid \lambda_{N}^{*}, M_{N}\right)$ is the maximized negative log-likelihood of training sequence set $O$. Then the optimum topology model $M_{N}{ }^{*}$ is

$$
M_{N}^{*}=\arg \min _{M_{N}}\left\{-2 \log P\left(O \mid \lambda_{N}^{*}, M_{N}\right)+K_{N} \log W\right\}
$$

We have proved that $P\left(O \mid \lambda_{N}^{*}, M_{N}\right)$ is a monotonously increasing function with respect to $N$, so the object function of (5) is a single peak function. We can use various optimum methods to solve (5), $e$. $g$. the golden section method.

\section{Data and Results}

\subsection{Data}




\begin{tabular}{|c|c|c|c|}
\hline Organism & Accession & Length & Web site \\
\hline SARS coronavirus BJ01 & AY278488. 2 & 221a.a. & $\begin{array}{l}\text { http: } / / \text { www. ncbi. nlm. nih. gov } / \text { entrez } / \text { query. fcgi? cmd = } \\
\text { Retrieve\&db }=\text { protein\&list }- \text { uids }=30275673 \& d o p t=\text { GenPept }\end{array}$ \\
\hline SARS coronavirus CUHK-W1 & AY278554.2 & 221a.a. & $\begin{array}{l}\text { http: } / / \text { www. ncbi. nlm. nih. gov } / \text { entrez } / \text { query. fcgi? } \mathrm{cmd}= \\
\text { Retrieve\&db }=\text { protein\&list }- \text { uids }=30023958 \& d o p t=\text { GenPept }\end{array}$ \\
\hline SARS coronavirus NC_004718.3 & NC_ -004718.3 & 221a.a. & $\begin{array}{l}\text { http: } / / \text { www. ncbi. nlm. nih. gov/entrez } / \text { query. fcgi? cmd = } \\
\text { Retrieve\&db }=\text { protein\&list-uids }=29836504 \& \text { dopt }=\text { GenPept }\end{array}$ \\
\hline SARS coronavirus urbani & AY278741.A & 221a.a. & $\begin{array}{l}\text { http: } / / \text { www. ncbi. nlm. nih. gov } / \text { entrez } / \text { query. fcgi? cmd }= \\
\text { Retrieve\&db }=\text { protein\&list }- \text { uids }=30027623 \text { \&dopt }=\text { GenPept }\end{array}$ \\
\hline Transmissible gastroenteritis virus & NC_ 002306.2 & 262a.a. & $\begin{array}{l}\text { http: } / / \text { www. ncbi. nlm. nih. gov/entrez/query. fcgi? cmd = } \\
\text { Retrieve\&db }=\text { protein\&list }- \text { uids }=13399294 \text { \&dopt }=\text { GenPept }\end{array}$ \\
\hline Human coronavirus $229 \mathrm{E}$ & $\mathrm{NC}_{-} 002645$ & 225a.a. & $\begin{array}{l}\text { http: } / / \text { www. ncbi. nlm. nih. gov/entrez/query. fcgi? } \mathrm{cmd}= \\
\text { Retrieve\&db }=\text { protein\&list-uids }=12175752 \& d \text { opt }=\text { GenPept }\end{array}$ \\
\hline Porcine-epidemic diarrhea virus & D49591 & 226a.a. & $\begin{array}{l}\text { http: } / / \text { www. ncbi. nlm. nih. gov } / \text { entrez } / \text { query. fcgi? } \mathrm{cmd}= \\
\text { Retrieve\&db }=\text { protein\&list }- \text { uids }=1360870 \& d o p t=\text { GenPept }\end{array}$ \\
\hline Bovine coronavirus & AF220295. 1 & 230a.a. & $\begin{array}{l}\text { http: } / / \text { www. ncbi. nlm. nih. gov } / \text { entrez } / \text { query. fcgi? cmd }= \\
\text { Retrieve\&db }=\text { protein\&list }- \text { uids }=17529680 \& d o p t=\text { GenPept }\end{array}$ \\
\hline Turkey coronavirus & JQ1172 & 230a.a. & $\begin{array}{l}\text { http: } / / \text { www. ncbi. nlm. nih. gov/entrez } / \text { query. fcgi? cmd }= \\
\text { Retrieve\&db }=\text { protein\&list }- \text { uids }=77083 \& d o p t=G e n P e p t\end{array}$ \\
\hline Avian-infectious bronchitis virus & M95169.1 & 225a.a. & $\begin{array}{l}\text { http: } / / \text { www. ncbi. nlm. nih. gov } / \text { entrez } / \text { query. fcgi? } \mathrm{cmd}= \\
\text { Retrieve\&db }=\text { protein\&dist }- \text { uids }=292958 \& d \text { opt }=\text { GenPept }\end{array}$ \\
\hline
\end{tabular}

\subsection{Results}

The multiple sequence alignment of the M protein produced by the ClustalW (1.8) software

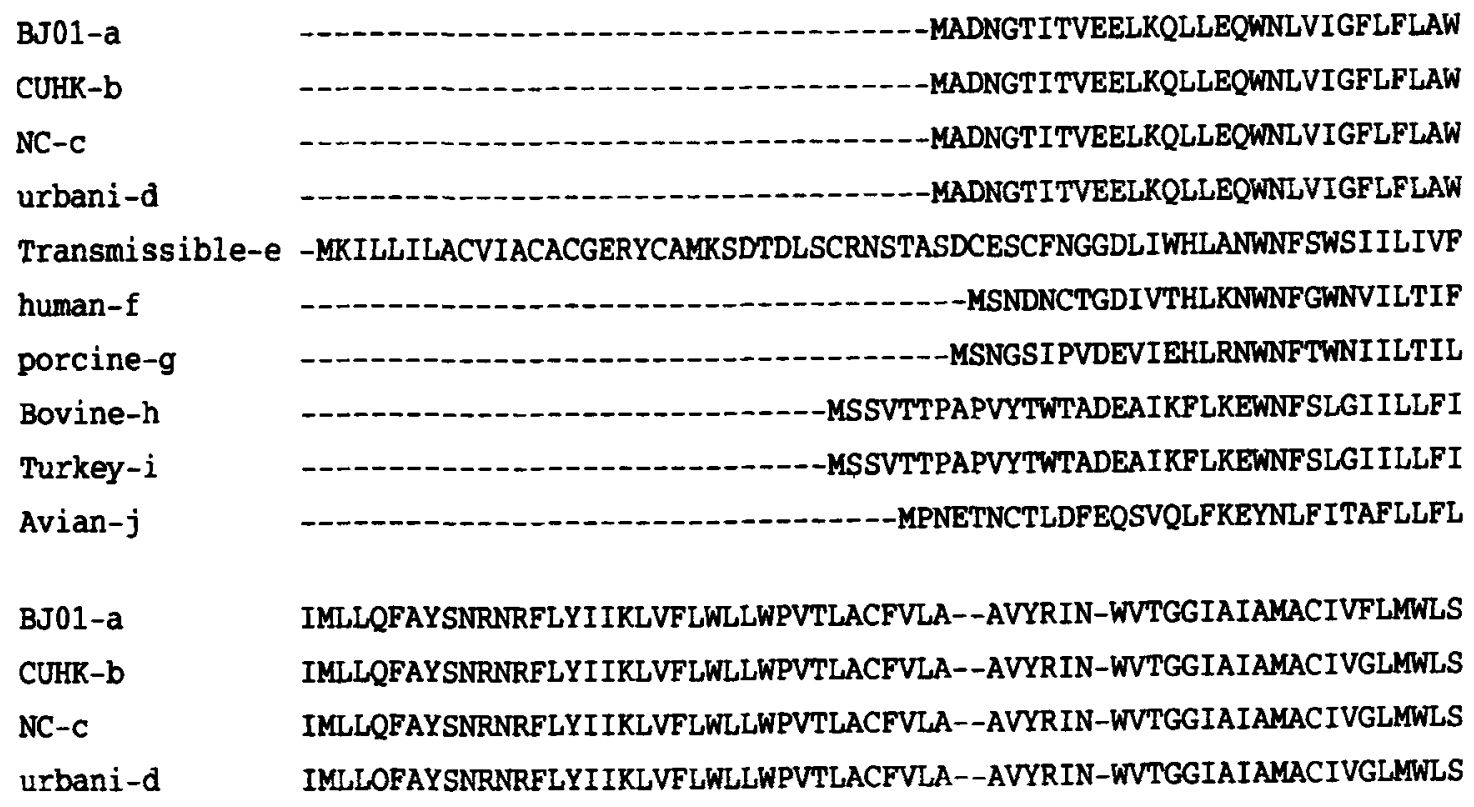


$\begin{array}{ll}\text { Transmissible-e } & \text { ITVLQYGRPQFSWFVYGIKMLIMWLLWVVLALTIFNAYSEYQVSRYVMFGFSIAGAIVTFVLWIM } \\ \text { human-f } & \text { IVILQFGHYKYSRLFYGLKMLVLWLWWPVLALSIFDTWANWDSN-WAFVAFSFFMAVSTLVWWVM } \\ \text { porcine-g } & \text { LVVLQYGHYKYSVFLYGVKMAILWILWPLVLALSLFDAWASFQVN-WVFFAFSILMACITLMLWIM } \\ \text { Bovine-h } & \text { TVILQFGYTSRSMFVYVIKMVILWLMWPLTIILTIFN--CVYALN-NVYLGFSIVFTIVAIIMWIV } \\ \text { Turkey-i } & \text { TIILQFGYTSRSMSVYVIKMIILWLMPLTIILTIFN--CVYALN-NVYLGFSIVFTIVAIIMWIV } \\ \text { Avian-j } & \text { TIILQYGYATRSKVIYTLKIVLWCFWPLNIAVGVIS--CTYPPN-TGGLVAIILTVFACLSFVG }\end{array}$

BJ01-a YFVASFRLFARTRSMWSFNPETNILLNVPLR-GTIVTRPLMESELVIGAVIIRGHLRMAGHSLGRCUHK-b YFVASGRLGARTRSMWSFNPETNILLNVPLR-GTIVTRPLMESELVIGAVIIRGHLRMAGHSLGRNC-c YFVASFRLFARTRSMWSFNPETNILLNVPLR-GTIVTRPLMESELVIGAVI IRGHLRMAGHSLGRurbani-d YFVASFRLFARTRSMWSFNPETNILLNVPLR-GTIVTRPLMESELVIGAVIIRGHLRMAGHPLGRTransmissible-e YFVRSIQLYRRTKSWWSFNPETKAILCVSAL-GRSYVLPLEGVPTGVTLTLLSGNLYAEGFKIAGG human-f YFANSFRLFRRARTFWAWNPEVNAITVTTVL-GQTYYQPIQQAPTGITVTLLSGVLYVDGHRLASG porcine-g YFVNSIRLWRRTHSWWSFNPETDALLTTSVM-GRQVCIPVLGAPTGVTLTLLSGTLLVEGYKVATG Bovine- $h$ YFVNSIRLFIRTGSWWSFNPETNNLMCIDMK-GRMYVRPI IEDYHTLTVTIIRGHLYMQGIKLGTG Turkey-i YFVNSIRLFIRTGSWWSFNPETNNLMCIDMK-GRMYVRPIIEDYHTLTVTI IRGHLYMQGIKLGTG Avian-j YWIQSIRLFKRCRSWWSFNPESNAVGSILLTNGQQCNFAIESVPMVLSPIIKNGVLYCEGQWLAK-

$\begin{array}{ll}\text { BJ01-a } & \text { CDIKDLPKEITVATSR-TLSYYKLGASQRVGTDSGFAAYNRYRIGNYKLNTDHAGSNDNIALLVQ-- } \\ \text { CUHK-b } & \text { CDIRDLPKEITVATSR-TLSYYKLGASQRVGTDSGFAAYNRYRIGNYKLNTDHAGSNDNIALLVQ-- } \\ \text { NC-c } & \text { CDIKDLPKEITVATSR-TLSYYKLGASQRVGTDSGFAAYNRYRIGNYKLNTDHAGSNDNIALLVQ-- } \\ \text { urbani-d } & \text { CDIKDLPKEITVATSR-TLSYYKLGASQRVGTDSGFAAYNRYRIGNYKLNTDHAGSNDNIALLVQ-- } \\ \text { Transmissible-e } & \text { MNIDNLPKYVYVALPSRTIVYTLVGKRLKASSATGWAYYVKSKAGDYSTEAR-TDNLSEQEKLLHMV } \\ \text { human-f } & \text { VQVHNLPEYMTVAVPSTTI IYSRVGRSVNSQNSTGWVFYRVKHGDFSAVSSPMSNMTENERLLHFF } \\ \text { porcine-g } & \text { VQVSQLPNFVTVAKATTTIVYGRVRSVNASSGTGWAFYVRSRHGDYSAVSNPSAVLTDSEKVLHLV } \\ \text { Bovine- } h & \text { YSLSDLPAYVTVAKVS-HLLTYKRGFLDKIGDTSGFAVYVSKVGNYRLPSTQKGSGLDTALLRNNI } \\ \text { Turkey-i } & \text { YSLSDLPAYVTVAKVS-HLLTYKRGFLDKIGDTSGFAVYVSKVGNYRLPSTQKGSGMDTALLRNNI } \\ \text { Avian- } j & \text { CEPDHLPKDIFVCTPDRRNIYRYVQKYTGDQSGNKKRFATFVYAKQSVDTGELESVATGGSSLYT-- }\end{array}$

The Multiple sequence alignment of the M protein produced by the SAHMM software

\begin{tabular}{|c|c|}
\hline J01-a & MADNGTI--- ------T VE-E-LKQLL EQWN----- ---LVI-GFLFLAWI----- \\
\hline CURK-b & MADNGTI-- - - \\
\hline $\mathrm{IC}-\mathrm{C}$ & -T VE-E-LKQLL EQWN-...-.-- --LVI-GFLFLAWI-..-- \\
\hline urbani-d & --T VE-E-LKQLL EQWN------ ---LVI-GFLFLAWI----- \\
\hline Transmissible-e & MKILLILACV IACACGERYC AM-K-SDTDL SCRNSTASDC ESCFNG-GDLIWHLANWNFS \\
\hline human- $\mathrm{f}$ & - T GD-I--VTHL KNWNF-- \\
\hline porcine-g & M-SNGSI-.- -..----P VD-E-VIEHL RNWNF--.-- ---TWN-IILTILLV--. \\
\hline Bovine- $h$ & MSSVTTPAPV YTW------T AD-E-AIKFL KEWNFS---- ---LGI--ILLFITV--- \\
\hline Turkey-i & MSSVTTPAPV YTW------T AD-E-AIKFL KEWNFS---- ---LGI--ILLFITI--- \\
\hline vian-j & --LFITAFLLFLTI-- \\
\hline
\end{tabular}




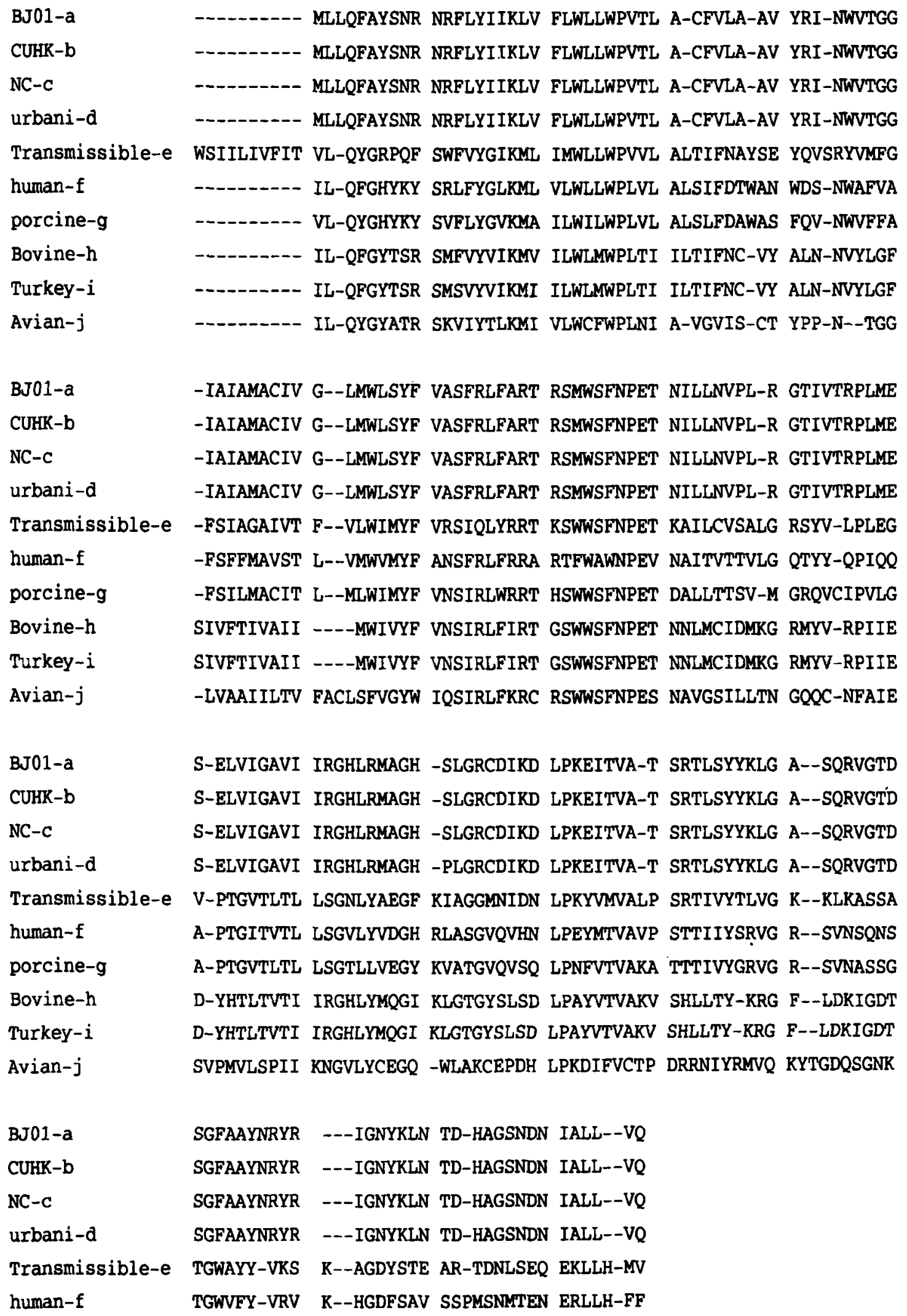




$\begin{array}{lll}\text { porcine-g } & \text { TGWAFY-VRS } & \text { K--HGDYSAV SNPSAVLTDS EKVLH-LV } \\ \text { Bovine-h } & \text { SGFAVY-VKS } & \text { K--VGNYRLP ST-QKGSGLD TALLRNNI } \\ \text { Turkey-i } & \text { SGFAVY-VKS } & \text { K--VGNYRLP ST-QKGSGMD TALLRNNI } \\ \text { Avian-j } & \text { KRFATF-VYA } & \text { KQSVDTGELE SV-ATGGS-- --SL--YT }\end{array}$

\section{References}

[1] Ksiazek T G, et al. A novel coronavirus associated with Severe Acute Respiratory Syndrome, The New England Journal of Medicine, 2003, April 10.

[2] Qin E' de, et al. A complete sequence and comparative analysis of a SARS-associated virus (isolate BJ01), Chi- nese Science Bulletin, 2003, 48(10): $941-948$.

[3] Durbin S, et al. Biological sequence analysis: Probabilistic models of proteins and nucleic acids, London: Cambridge University Press, 1998.

[4] Schwarz G. Estimating the dimension of a model, Annuals of Statistics, 1978, 6: 461-464.

(Executive editor SHEN Mei-Fang) 